\title{
Thermal-magnetic relation in a sunspot and a map of its Wilson depression
}

\author{
S. K. Mathew ${ }^{1,2}$, S. K. Solanki ${ }^{1}$, A. Lagg ${ }^{1}$, M. Collados ${ }^{2}$, J. M. Borrero ${ }^{1}$, and S. Berdyugina ${ }^{3}$ \\ 1 Max-Planck-Institut für Aeronomie, 37191 Katlenburg-Lindau, Germany \\ e-mail: [solanki; lagg; borrero]@linmpi .mpg.de \\ 2 Instituto de Astrofísica de Canarias, La Laguna, Tenerife, Spain \\ e-mail: shibu;mcv@ll.iac.es \\ 3 Institut für Astronomie, ETH, 8092 Zürich, Switzerland \\ e-mail: sveta@astro.phys.ethz.ch
}

Received 22 July 2003 / Accepted 31 March 2004

\begin{abstract}
We present relations between thermal and magnetic quantities in a simple, isolated sunspot, as deduced from the inversion of $1.56 \mu \mathrm{m}$ spectropolarimetric data. We used a combination of two infrared Fe I lines at $15648.5 \AA$ and $15652.8 \AA$ in the inversions. Due to the high Zeeman sensitivity of these lines, we can study this relationship in the entire sunspot. The relevant parameters were derived both as a function of location within the sunspot and of height in the atmosphere using an inversion technique based on response functions. In this paper we relate the magnetic vector with temperature. We find a nonlinear relationship between the various components of the magnetic vector and temperature, which confirm the results from earlier investigations. We also computed the Wilson depression and the plasma $\beta$ for the observed sunspot and compare our results with earlier findings.
\end{abstract}

Key words. sunspots - Sun: magnetic fields - Sun: infrared

\section{Introduction}

A relationship between temperature and magnetic field in a sunspot has been theoretically predicted and computed (Alfvén 1943; Maltby 1977; Dicke 1970) and has been studied observationally (Kopp \& Rabin 1992; Solanki et al. 1993; Martínez Pillet \& Vázquez 1993; Stanchfield et al. 1997; Westendorp Plaza et al. 2001; Penn et al. 2002, 2003). Observations support a linear relationship between the squared field strength and temperature in the umbra, whereas a strong departure from the predicted linear relationship is seen outside the umbra (Kopp \& Rabin 1992; Solanki et al. 1993; Balthasar $\&$ Schmidt 1994). A smooth transition in field strength is observed near the umbral-penumbral boundary, whereas the continuum intensity and hence the temperature displays a jump near this boundary (see Solanki 2003 for a review). The difference between the predicted and observed behavior suggests that the measurements do not sample the same layers in the umbra and penumbra.

Since in the sunspots the observed relationship between the magnetic field and temperature must be a consequence of the magnetostatic horizontal force balance, the relationship between $B_{z}, B_{r}$ and the gas pressure $P_{\mathrm{g}}$ is given by,

$P_{0}(z)=P_{\mathrm{g}}(r, z)+B_{z}^{2}(r, z) / 8 \pi+F_{\mathrm{c}}(r, z) / 8 \pi$,
$F_{\mathrm{c}}(r, z)=2 \int_{r}^{a} B_{z}\left(r^{\prime}, z\right) \frac{\partial B_{r}\left(r^{\prime}, z\right)}{\partial z} \mathrm{~d} r^{\prime}$.

Here, $P_{0}(z)$ is the quiet Sun gas pressure, $P_{\mathrm{g}}$ is the gas pressure inside the sunspot and $B_{z}$ and $B_{r}$ are the vertical and radial field components, respectively. $a$ is the radial distance of the sunspot boundary, $r$ and $z$ are the radial and vertical coordinates, and $F_{\mathrm{c}}$ represents the curvature integral. Martínez Pillet \& Vázquez (1993) were able to utilize this relationship for calculating the Wilson depression under the assumption of known curvature force. They computed a Wilson depression of 400-800 km in the umbra of their observed sunspots (with the range being given by different assumed values of $F_{\mathrm{c}}$ ), which agrees well with previous determinations (Gokhale \& Zwaan 1972). Solanki et al. (1993) used a similar technique and estimated the Wilson depression as a function of radial distance in a sunspot. They found that the $\tau=1$ level is depressed by around $100-150 \mathrm{~km}$ in the penumbra and $400-450 \mathrm{~km}$ in the umbra under the assumption that the integrated radial curvature force $F_{\mathrm{c}}=0$.

Here we describe an investigation of the thermal-magnetic relation in a simple sunspot based on parameters obtained from an inversion of spectral data obtained in two adjacent $\mathrm{Fe}$ I lines in the IR H-band (Mathew et al. 2003). Due to the superior 

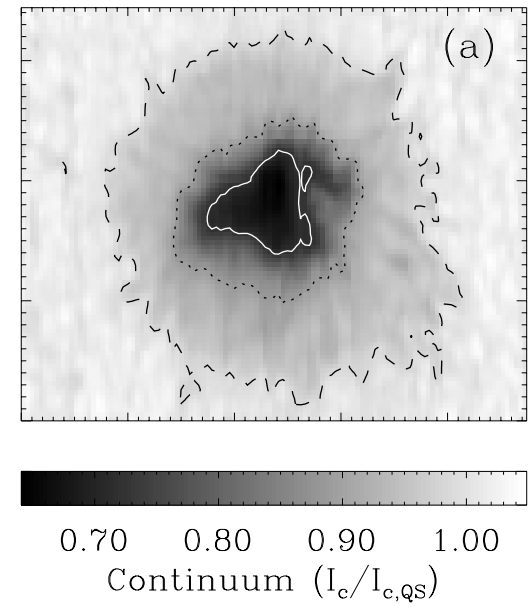
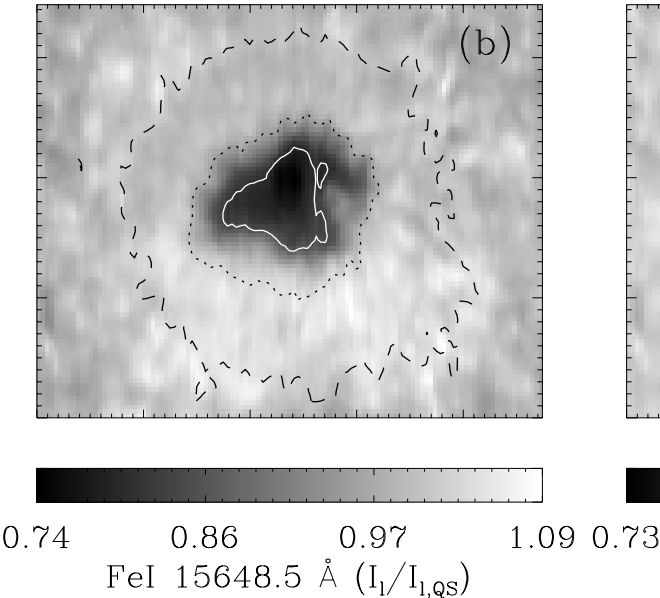
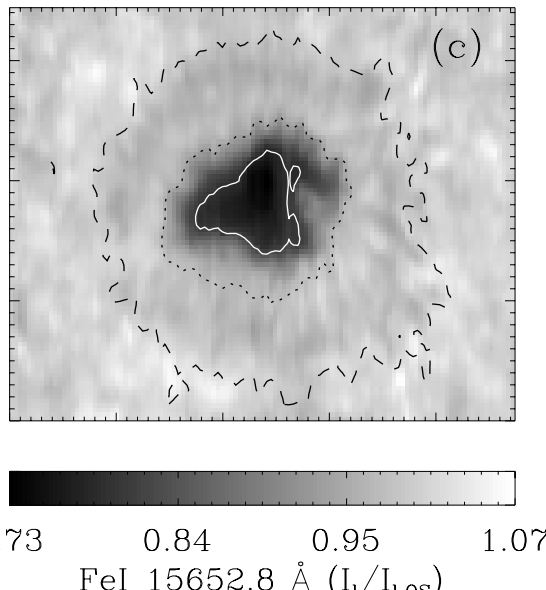

Fig. 1. Continuum a) and line core intensity b) and c) images of the observed sunspot. The contours mark the boundaries between three continuum temperature zones, which correspond to the inner umbra (bounded by solid white), the umbral-penumbral boundary (between solid and dotted dark), and the penumbra (between dotted and dashed dark lines). The displayed intensities are normalized to the average quiet sun value. The spectrograph slit is placed in the vertical direction and the spot is scanned from left to right. Each tick mark corresponds to $1^{\prime \prime}$.

Zeeman sensitivity of Fe I $15648.5 \AA$ line (and partly the inversion of molecular lines simultaneously with atomic lines) it is possible to investigate the thermal-magnetic relationship in the whole sunspot (Solanki et al. 1993). Also, the effect of stray light is smaller in the infrared, which otherwise could contaminate the intensity profiles (Kopp \& Rabin 1992). Since a map of the magnetic and thermal properties over the whole sunspot is available, we determine a map of the Wilson depression of this spot and also the plasma $\beta$ in it. Finally we compare our results with earlier findings obtained using visible and infrared lines.

\section{Data and analysis}

We use spectropolarimetric data (full Stokes profiles), simultaneously recorded on 27th Sep 1999, in two IR Fe I lines (15648.5 $\AA, g=3$ and $15652.8 \AA, g$ eff $=1.53)$ with the TIP (Tenerife Infrared Polarimeter, Collados 1999; Martínez Pillet et al. 1999). A scan was made of a fairly round sunspot, when it was near the disk center $(\mu=0.92)$. The spot belongs to active region NOAA 8706 and had a diameter of around $31^{\prime \prime}$. Figure 1 shows the normalized continuum and line core intensity images of the observed sunspot.

Continuum intensities are obtained from the mean values of Stokes $I$ profiles over a line-free window near $15646.7 \AA$. The continuum intensity, $I_{\mathrm{c}, \mathrm{QS}}$, averaged over all the points where the polarization signal integrated over the observed spectral range $P=\left[\left(Q^{2}+U^{2}+V^{2}\right) / I^{2}\right]^{1 / 2}<10^{-3}$ has been employed to normalize the intensity. Contours in the figure represent three continuum intensity zones which approximately correspond to umbra, umbral-penumbral boundary and the penumbra in the intensity histogram distribution.

In Fig. 1 the slit is placed in the vertical direction and the spot is scanned from left to right. The observations were carried out with a spectral sampling of $29 \mathrm{~m} \AA$, and covered a spectral range of $7 \AA$. The good and uniform seeing conditions during the observing interval kept the image blurring low. From the average photospheric power spectrum, we estimated the spatial resolution to be in the range of $\sim 1^{\prime \prime}-1.2^{\prime \prime}$. We fit straight lines to the flat and steadily increasing parts in the power spectrum, respectively. The intersection of these two straight lines provides the cutoff spatial frequency which is taken as the seeing limit. The correlation tracker installed at the VTT (Ballesteros et al. 1996) was used to stabilize the image, which allowed a smooth scanning throughout the observing run. Line core intensities are obtained by detecting the minimum of the respective absorption lines. In order to accurately determine the minimum, we used a polynomial fit about the pixel with the lowest intensity within the absorption line at a given spatial point. The line core quiet Sun intensity $I_{1, \mathrm{QS}}$ is obtained by the same method as described for the continuum intensity. Figure 2 shows sample Stokes $I$ profiles for a disk side (solid line) and a limb side (dashed line) penumbral point. The shift between the two plotted profiles is due to the Evershed effect. The difference in line core intensity in the disk side and limb side penumbral profiles is attributed to a difference in inclination of the field and is discussed in more detail in Sect. 4. The data have been described in greater detail by Mathew et al. (2003, hereafter Paper I).

The data were inverted by Mathew et al. (2003) using the code "SPINOR" described by Frutiger et al. (2000). This code incorporates the "STOPRO" routines (Solanki 1987), which compute synthetic Stokes profiles of one or more lines upon input of their atomic data and one or more model solar atmospheres. LTE (Local Thermodynamic Equilibrium) conditions are assumed and the Unno-Rachkovsky radiative transfer equations (RTE) are solved. Starting with an initial guess model, the synthetic profiles were iteratively fit to the observed data using response functions (RFs) and the LevenbergMarquardt (Press et al. 1992) algorithm to minimize the merit function $\chi^{2}$ (Ruiz Cobo \& del Toro Iniesta 1992; Frutiger 2000). The inversion returned temperature $(T)$, magnetic field strength $(B)$, field inclination $(\gamma)$, field azimuth $(\chi)$, line-ofsight velocity $\left(V_{\mathrm{LOS}}\right)$ stratifications, as well as micro- $\left(\xi_{\text {mic }}\right)$ and macro-turbulent $\left(\xi_{\mathrm{mac}}\right)$ velocities. Using the known location of 

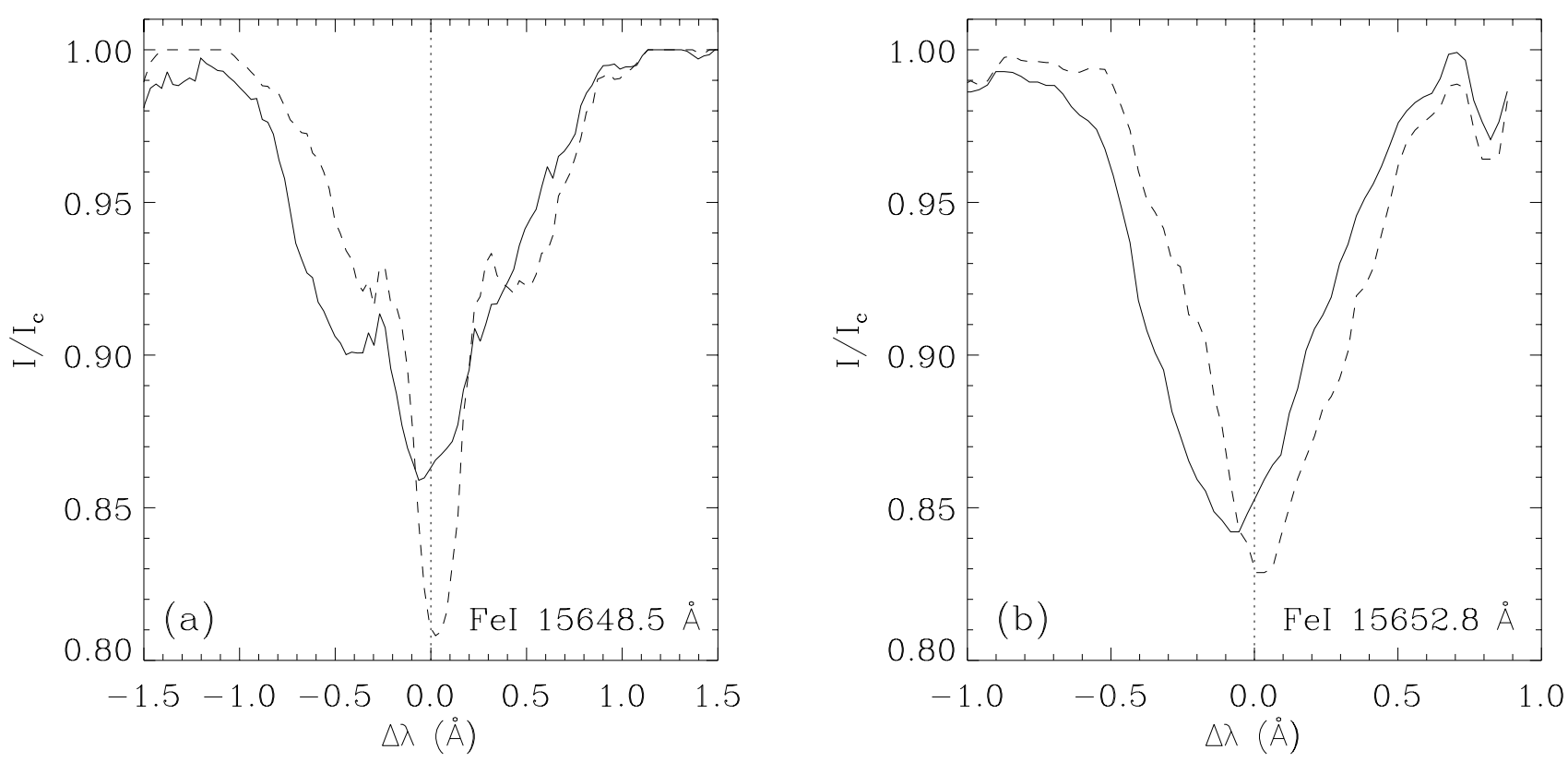

Fig. 2. Sample profiles of Fe I 15648.5 a) and $15652.8 \AA$ b) line from the disk side (solid curve) and limb side (dashed curve) penumbra.

the sunspot, $\gamma$ and $\chi$ are converted into angles relative to the solar coordinates. Of interest here is the zenith angle $\zeta$. It is used to determine $B_{z}$, the vertical component of the magnetic field. More details on this particular data set, the inversion procedure and the results have been given in Paper I.

For this investigation the continuum temperature, obtained by converting continuum intensity into temperature using the Planck function (Solanki et al. 1993) is employed. We convert the continuum intensity into temperature solving the following equation for $T$,

$\frac{I_{\mathrm{c}}}{I_{\mathrm{c}, Q S}}=\frac{\mathrm{e}^{h c / \lambda k_{\mathrm{B}} T_{0}}-1}{\mathrm{e}^{h c / \lambda k_{\mathrm{B}} T}-1}$.

Here, $h$ is Planck's constant, $k_{\mathrm{B}}$ is Boltzmann's constant, $c$ is the speed of light, $I_{\mathrm{c}} / I_{\mathrm{c}, \mathrm{QS}}$ is the normalized continuum intensity with respect to the quiet $\operatorname{Sun}, T_{0}$ is the quiet Sun temperature and $\lambda$ is the wavelength. We adopt a value for $T_{0}\left(\tau_{1.6}=1\right)=$ $7058 \mathrm{~K}$ taken from the quiet Sun model described by Maltby et al. (1986). Instead of using the temperature returned from inversions, we stick to this choice, because of the poor sensitivity of these lines to the temperature, due to the telluric and molecular blends. In the following sections, we mainly concentrate on the thermal-magnetic relation for deep atmospheric layers, specifically we consider the layer averaged over $\log \tau_{1.6}$ values ranging from 0 to -0.5 .

\section{Stray light considerations}

Stray light can affect the relation between magnetic parameters and temperature (Solanki et al. 1993; Martínez Pillet \& Vázquez 1993). In order to take care of stray light, in our inversions the Stokes profiles were calculated in LTE through a two component model atmosphere: in every pixel two atmospheric components are allowed for, one magnetic (with a filling factor $\alpha$ ) and one field-free (with filling factor $1-\alpha$ ), where $\alpha$ is a free parameter of the inversions (Paper I). The filling factor $1-\alpha$ of the field-free component is a measure of the stray light contamination in the profiles. Note that any emission from field-free gas within the solar surface area sampled by a pixel is formally counted to the stray light. In Fig. 16 of Paper I we plot the azimuthal averages for the continuum intensity $\left(I_{\mathrm{c}} / I_{\mathrm{c}, \mathrm{QS}}\right)$ and the scattered light parameter $(1-\alpha)$ for the analyzed sunspot. The average scattered light parameter is less than 0.1 in the umbra and increases to $\sim 0.5$ near the outer penumbra. A local maximum in this parameter is noticed near the umbral-penumbral boundary, where the gradient in continuum intensity is large.

Here we use the parameter $\alpha$ to correct the continuum intensity in the following way. The observed continuum intensity $I_{\mathrm{obs}}$ can be written as,

$I_{\mathrm{obs}}=\alpha I_{\text {spot }}+(1-\alpha) I_{\text {scat }}$

where $I_{\text {spot }}$ is the real continuum intensity emerging in the spot and $I_{\text {scat }}$ is the stray light contamination from the surroundings. We assume that $I_{\text {scat }}$ basically originates from the quiet Sun photosphere and assign the average quiet Sun continuum intensity value to $I_{\text {scat }}$. From Eq. (4) we then obtain an estimate of $I_{\text {spot }}$, which we then use to derive the temperature. This eliminates to first order the effect of stray light in our derived thermal-magnetic relations. Second order effects, such as polarized scattered light from the penumbra in the umbra still remains, however. For a comparison, in Fig. 3 we plot the temperature derived from the continuum intensity before and after the stray light correction. The dashed line represents slope unity. Evidently, stray light introduces an increase in the derived temperature, as expected. 


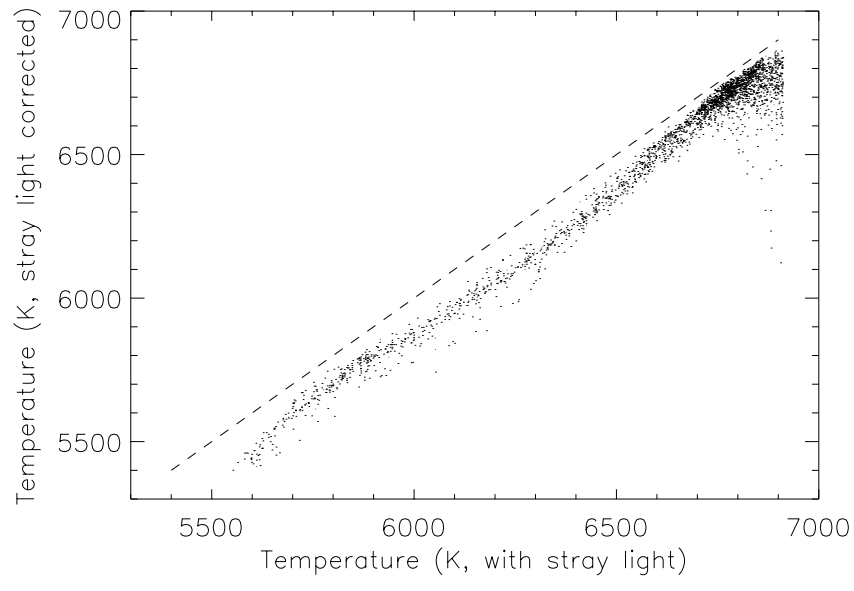

Fig. 3. Computed continuum temperature before and after the stray light correction. The dashed line marks exact equality between the two quantities.

\section{Observed relationships}

Figure 4a shows the $B$ vs. $T$ relationship for optical depths averaged over $\log \tau_{1.6}$ from 0 to -0.5 for all the points within the sunspot. The open circles denote the umbral points, the plus signs umbral-penumbral transition region and the triangles the penumbral points. The difference in slope between the umbra, umbral boundary and penumbra is clearly visible. Our result is qualitatively similar to the results obtained by earlier investigators, quantitative differences are expected to arise largely from different heights sampled. We employ the average over the log $\tau_{1.6}$ range 0 to -0.5 of the parameters returned by the inversion in order to take into account somewhat conflicting requirements that on the one hand the magnetic and thermal quantities should if possible refer to the same height (i.e. around $\log \tau_{1.6}=0$ ) while on the other hand the deduced magnetic field is more reliable where the employed spectral lines obtain a large contribution (i.e. around $\log \tau_{1.6}=-1$ to -0.5 ).

In the umbra, the magnetic field strength decreases with increasing temperature and the distribution is distinctly nonlinear. For the umbra (as defined for the purposes of this paper), two regions can be identified in the plot, a strong variation of the field strength with temperature below $5700 \mathrm{~K}$ and a gradual change in the field strength with temperature between 5700 and $5900 \mathrm{~K}$. The penumbral distribution of $B$ versus $T$ shows a strong overall decrease of $B$ with increasing $T$, with an increased scatter. A gradual change in field strength is found for the umbral-penumbral transition region. The break between the $B-T$ relation in the umbra and in the umbra-penumbra region become more clearly visible if we take $B$ from the higher layer ( e.g. $\log \tau_{1.6}=-0.5$ or -1.0 ). Then the deduced curves look more similar to those plotted by Kopp \& Rabin (1992) and Solanki et al. (1993). The height averaged field strength they measured refers to a height with large response function of the spectral line to magnetic field strength, which is at around $\log \tau_{1.6}=-0.5$ to -1.0 .

Figure $4 \mathrm{~b}$ shows a field zenith angle $\zeta$ versus $T$ diagram averaged over the same $\log \tau$ layers. As in the case of $B, \zeta$ also displays a nonlinear relationship with $T$. $\zeta$ remains below roughly $30^{\circ}$ throughout the umbra, and the variation with temperature is smooth across the umbral-penumbral boundary. Towards the higher temperature region (i.e. in the penumbra) a steep increase in $\zeta$ is noticed. The points with $\zeta>90^{\circ}$ (i. e. those lying above the dotted line in Fig. 3b) imply return flux. They coincide with down flows in the outer penumbra (Paper I).

In Figs. $4 \mathrm{c}$ and $4 \mathrm{~d}$ we plot the vertical $\left(B_{z}\right)$ and radial $\left(B_{r}\right)$ field components versus $T$. Note that the relation between $\zeta$ and temperature influence the $B_{z}$ and $B_{r}$ versus $T$ relation. $B_{z}$ shows a similar trend as the field strength $B$, except that there is practically no break between the inner umbra and the umbral boundary layer. Also, as expected, $B_{z}$ drops almost to zero at the highest temperatures. It actually drops slightly below zero at locations where $\zeta$ runs beyond $90^{\circ}$ near the penumbral boundary. $B_{r}$, on the other hand, increases over the whole temperature range, although the exact relation is masked by the large scatter. The relatively small variations of the magnetic parameters across the umbra-penumbra boundary basically describes the fact that this boundary is relatively narrow (see Fig. 1) and the field changes gradually with radial distance. Similarly the steep change of the magnetic field in the penumbra only signifies that the temperature is relatively homogeneous there.

In addition to the thermal-magnetic relation, we also obtained a plot for field zenith angle $\zeta$ versus $B$ which is shown in Fig. 6a. The results are similar to the one obtained by Stanchfield et al. (1997). Here the relationship is almost linear, with a slight S-shape and a large scatter near the umbral penumbral boundary. Much of the scatter is introduced by the larger uncertainty in the results obtained from inversion at $\log \tau_{1.6}=0$. For comparison in Fig. 6b we plot field strength versus zenith angle at $\log \tau_{1.6}=-0.5$. An anti correlation is now clearly visible. This figure explains the similarity between the thermal-magnetic relationship involving $B$ and $\zeta$ (Figs. $4 \mathrm{a}$ and $4 \mathrm{~b}$ ) and the much steeper dependence of $B_{z}$ on temperature (Fig. 4c) than of $B$ (Fig. 4a).

Figures $5 \mathrm{a}$ and $5 \mathrm{~b}$ show scatter plots of field strength $(B)$ versus core intensity in the Fe I 15648.5 and $15652.8 \AA$ lines, respectively. The shape of the core intensity plot differs from the continuum $B$ vs. $T$ plot especially at higher temperatures. The observed relationship between the magnetic field strength and line core intensity is similar to the results presented by Stanchfield et al. (1997). Intriguing is that the Fe I $15648.5 \AA$ points in the core intensity plot follow two distinct paths in the penumbra. The $15652.8 \AA$ line also shows rudimentary signs of such a behavior. Further analysis reveals that the two branches are populated by disk side and limb side penumbral points. The property of relevance for this behavior is the difference in inclination of the field with respect to the lineof-sight. For larger field inclinations (as is the case for limb side penumbral points), the peak ratio of the Zeeman $\pi$ to $\sigma$ components becomes larger, which results in a reduction of the line core intensity. This effect is more prominent in a completely Zeeman split line, such as Fe I $15648.5 \AA$ A. A noticeable difference in core intensity is seen in the disk side $\left(\gamma \sim 4^{\circ}\right)$ and limb side $\left(\gamma \sim 80^{\circ}\right)$ penumbral Fe I $15648.5 \AA$ intensity profiles shown in Fig. 2a. Both correspond to otherwise similar parameters $(B \sim 1700 \mathrm{G}, T \sim 6200 \mathrm{~K})$. The less Zeeman sensitive Fe I $15652.8 \AA$ line, plotted in Fig. 2b, exhibits a far smaller 

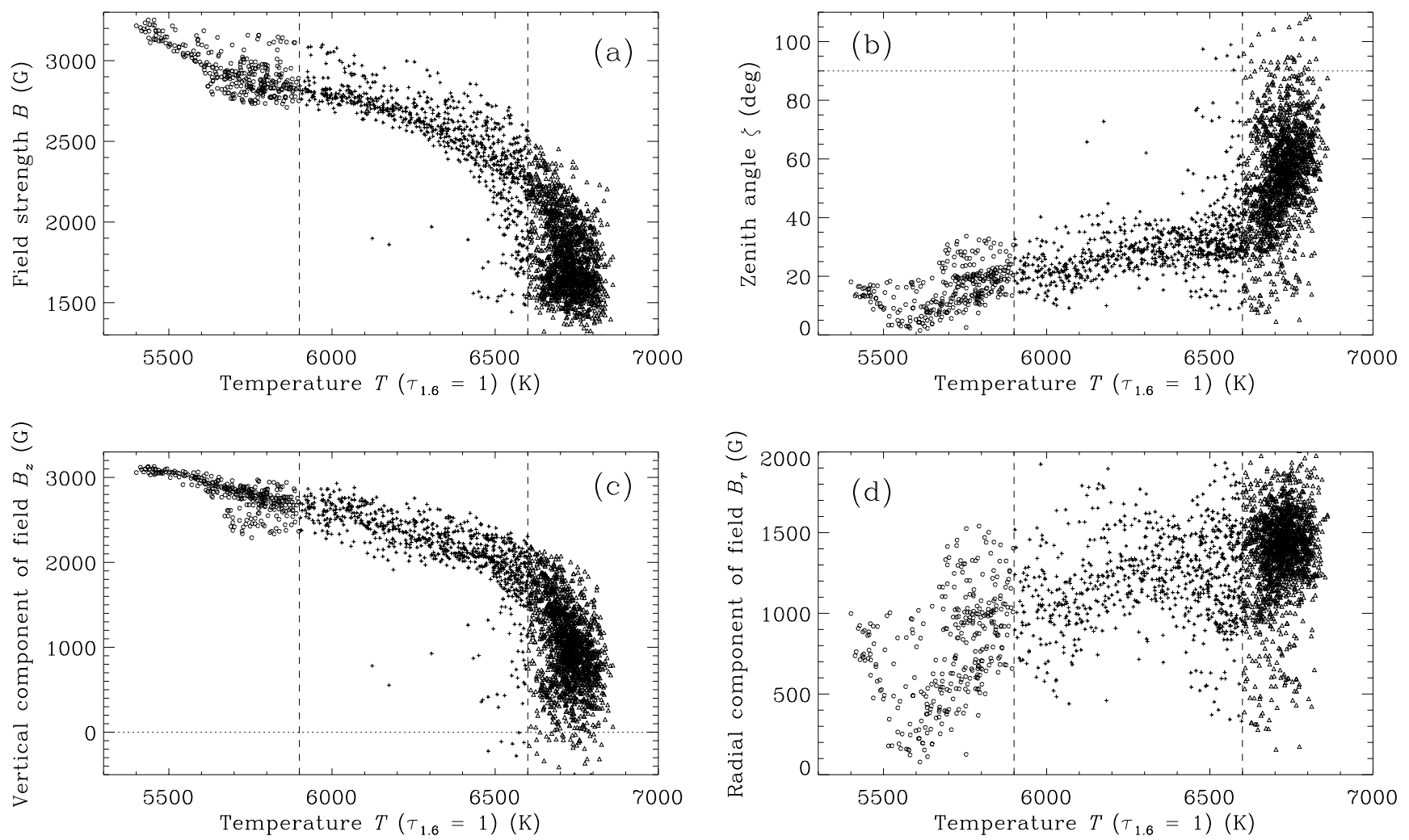

Fig. 4. Magnetic field strength $B$ a), zenith angle $\zeta \mathbf{b}$ ), Magnetic field components $B_{z} \mathbf{c}$ ) and $B_{r}$ d) versus continuum brightness temperature $T$. The circles denote the umbral points, the plus signs represent points in the umbral-penumbral boundary region, and the triangles the penumbral points. The vertical dashed line indicate the temperature corresponding to the inner and middle contours in Fig. 1.
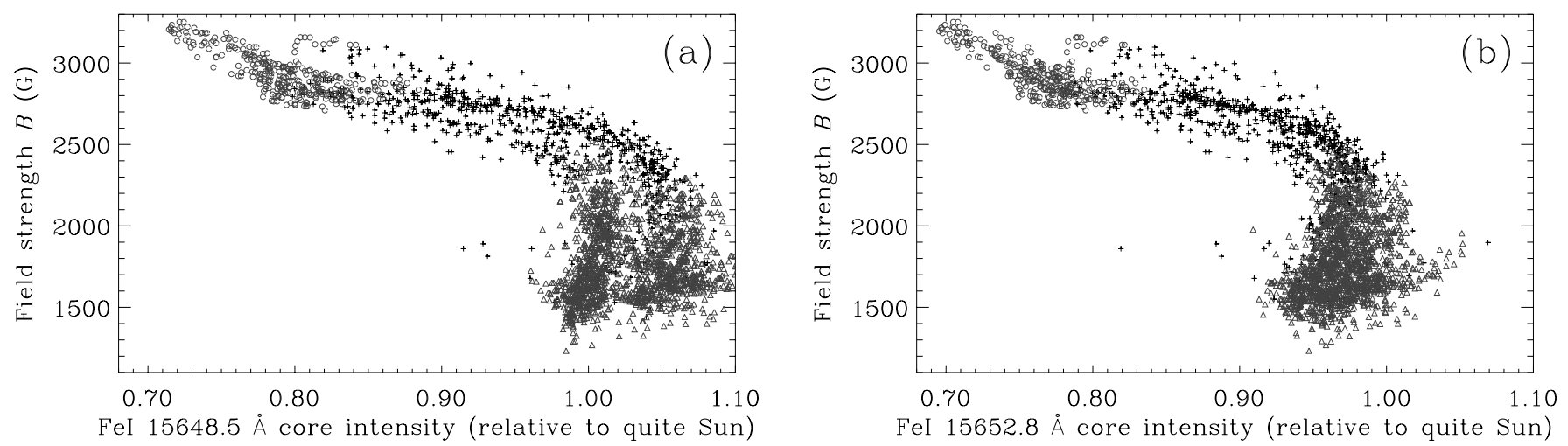

Fig. 5. Magnetic field strength $B$ versus FeI 15648.5 a) and $15652.8 \AA$ b) core intensity. The symbols have the same meaning as in Fig. 4.
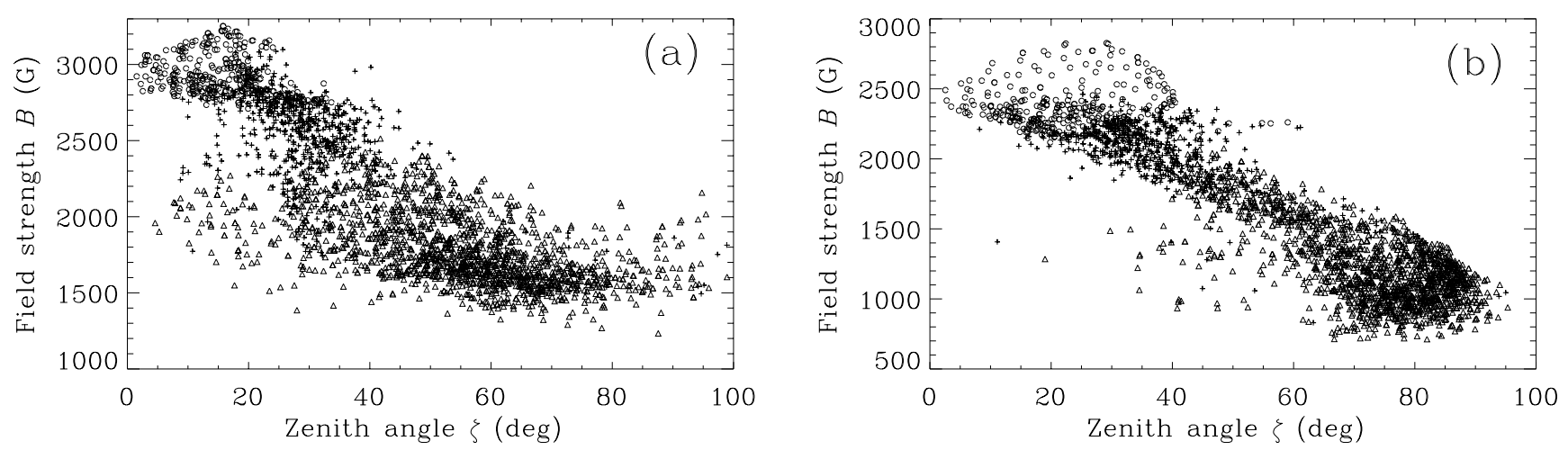

Fig. 6. Magnetic field strength $B$ versus field zenith angle $\zeta$, a) averaged over $\log \tau_{1.6}=0$ to -0.5 , b) for the layer at $\log \tau_{1.6}=-0.5$. The symbols have the same meaning as in Fig. 4. 




Fig. 7. Wilson depression $\left(Z_{\mathrm{W}}\right)$ of the observed sunspot. The inner and outer contours represents the umbral and penumbral boundaries obtained from the continuum image, respectively.

difference (the shift between the two plotted profiles is due to the Evershed effect).

\section{Wilson depression and plasma $\beta$}

In this section we present maps of the Wilson depression $\left(Z_{\mathrm{W}}\right)$, gas pressure $\left(P_{\mathrm{g}}\right)$, magnetic pressure $\left(P_{\mathrm{B}}\right)$ and plasma $\beta(=$ $\left.8 \pi P_{\mathrm{g}} / B^{2}\right)$ for the observed sunspot. The Wilson depression is obtained by a method described by Solanki et al. (1993) cf. Martínez Pillet \& Vázquez (1993). The relationship between $B_{z}, B_{r}$ and gas pressure $P_{\mathrm{g}}$ in the sunspot can be derived by the integration of the radial component of the magnetostatic horizontal force balance equation in the sunspot and is given in Eq. (1). The gas pressure within the sunspot is eliminated by introducing the equation of state, and Eq. (1) can be re-written as,

$\frac{T(r, z)}{T_{0}(z)}=\frac{m(r, z)}{m_{0}(z)} \frac{\rho_{0}(z)}{\rho(r, z)}\left[1-\frac{B_{z}^{2}(r, z)+F_{\mathrm{c}}(r, z)}{8 \pi P_{0}(z)}\right]$,

where $\rho$ is the gas pressure, and $m$ the mean molecular weight. The second term (magnetic) in Eq. (5) is the sum of two components, the first one $\left(B_{z}^{2}\right)$ is associated with the magnetic pressure and the second $\left(F_{\mathrm{c}}\right)$ with the horizontal tension forces due to the bending of the field lines. Martínez Pillet \& Vázquez (1993) proposed that the above equation could be utilized to obtain an estimate of the Wilson depression, $Z_{\mathrm{W}}$, which can be defined as,

$Z_{\mathrm{W}}(r, \lambda)=z\left(\tau_{0.5}=1, \mathrm{QS}\right)-z\left(\tau_{\lambda}=1, r\right)=-z\left(\tau_{\lambda}=1, r\right)$,

where $\tau_{\lambda}$ is the continuum optical depth at wavelength $\lambda$ and $z$ increases in the direction of decreasing $\tau$. Above we have set $z\left(\tau_{0.5}=1\right)=0$ in quiet Sun. Using Eq. (5), the total pressure in the sunspot, $P_{0}(z)$, can be determined from the measured $B$ and $T$ in the sunspot. A comparison of $P_{0}$ with a standard model of the quiet atmosphere and convection zone can be used to fix the height at which $B$ and $T$ are determined, this depth is approximately equal to the Wilson depression $Z_{\mathrm{W}}$.
Martínez Pillet \& Vázquez (1993) rewrite Eq. (5) as,

$$
\begin{aligned}
\frac{T(r, z)}{T_{0}(z=0)}= & \frac{m(r, z)}{m_{0}(z)} \frac{\rho_{0}(z)}{\rho(r, z)} \frac{T_{0}(z)}{T_{0}(z=0)} \\
& \times\left[1-\frac{1+f(r, z)}{8 \pi P_{0}(z)} B^{2}(r, z)\right]
\end{aligned}
$$

where,

$f(r, z)=\frac{F_{\mathrm{c}}(r, z)-B_{r}^{2}(r, z)}{B^{2}(r, z)}$.

Assuming,

$\frac{m(r, z)}{m_{0}(z)} \frac{\rho_{0}(z)}{\rho(r, z)} \frac{T_{0}(z)}{T_{0}(z=0)}=$ constant

in the umbra, and assigning a constant value for $f(r, z)$ for all the points in the umbra, they obtain values for $Z_{\mathrm{W}}$ between $440-760 \mathrm{~km}$ for $0 \leq f \geq 1$.

Since we have the information on gas pressure $P_{\mathrm{g}}$ and $B_{z}$ for each spatial position in the sunspot from our inversions, we use these values in order to compute the total pressure $P_{0}(x, y, z)$ at each location in the sunspot. We use Eq. (1) for our computations. While deriving $Z_{\mathrm{W}}$, we kept $F_{\mathrm{c}}=0$, since this is the simplest assumption to make. The value of geometrical height $z$, corresponding to the computed pressure $P_{0}$ is obtained from a standard quiet Sun model. For the present purpose we use Spruit's (1974) model, which combines a model of the convection zone with an empirical model atmosphere (HSRA; Gingerich et al. 1971).

In Fig. 7 we plot the $z\left(\tau_{1.6}=1\right)=-Z_{\mathrm{W}}$ surface for the observed sunspot. The bottom plane in the plot shows the corresponding image of the Wilson depression. The white contours in the image represent the umbral and the penumbral boundaries obtained from the continuum intensity image. We obtained average values for $Z_{\mathrm{W}}$ of close to $400 \mathrm{~km}$ in the umbra and around $120 \mathrm{~km}$ for the penumbra. A jump in $Z_{\mathrm{W}}$, corresponding to the steep variation in temperature is seen across the umbral-penumbral boundary. 

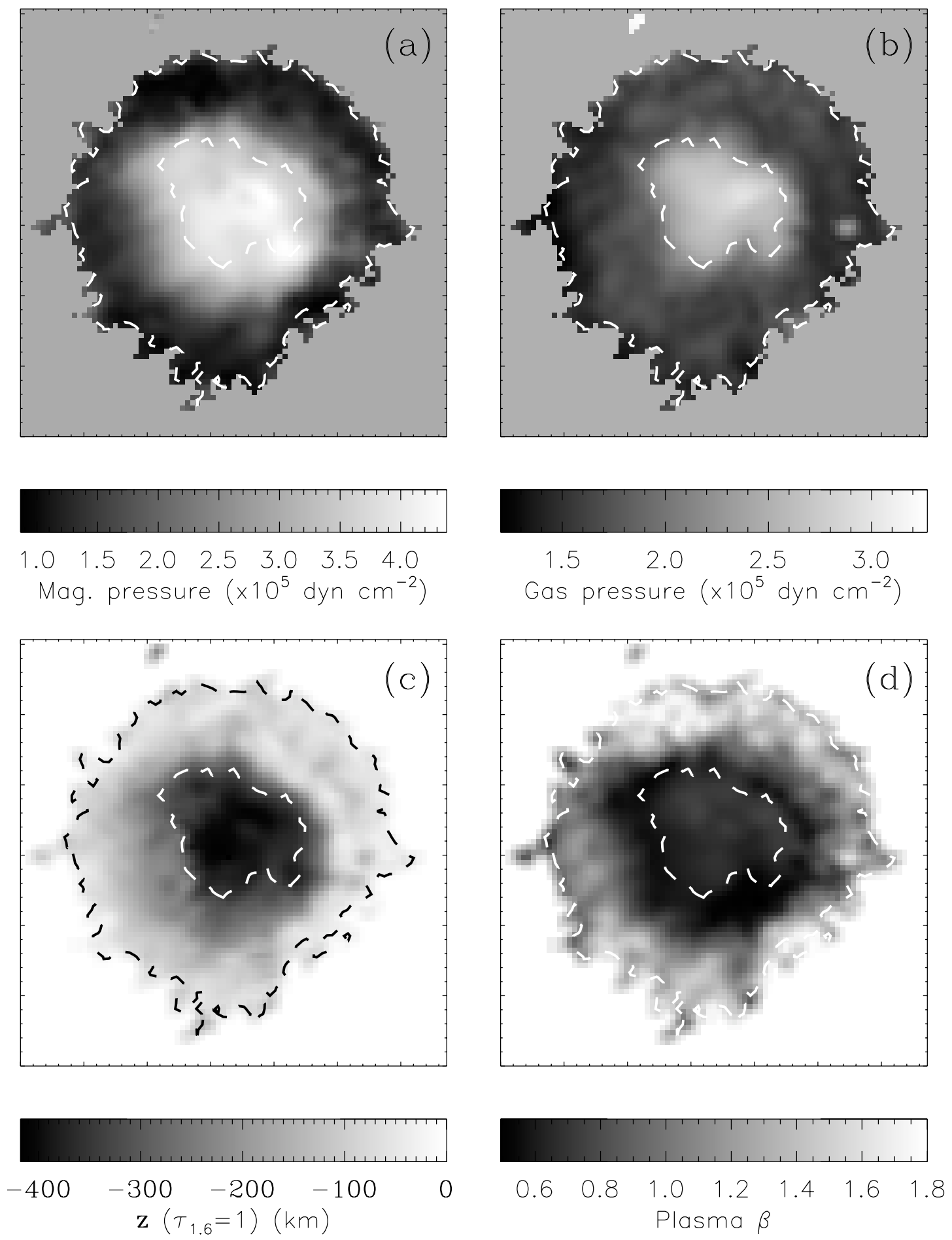

Fig. 8. Magnetic pressure a), gas pressure b), Wilson depression c), and the plasma $\beta$ d) for the observed sunspot. The inner and outer contours represents the umbral and penumbral boundaries obtained from the continuum image, respectively.

In Figs. 8a-d we plot maps of the magnetic pressure, gas pressure, Wilson depression, and plasma $\beta$, respectively. The magnetic pressure is computed from the magnetic field strength, i.e. $P_{\mathrm{B}}=B^{2} / 8 \pi$. In the umbra and at least in the inner penumbra, the magnetic pressure $P_{\mathrm{B}}$ is larger than the gas pressure $P_{\mathrm{g}}$. Gas pressure varies drastically across the umbralpenumbral boundary, whereas the magnetic pressure variation is steep in the outer penumbra. There the magnetic pressure actually drops below the gas pressure. This can be seen more clearly in Fig. 8d, where we display the plasma $\beta . \beta$ is less than unity in most of the umbra and in the inner part of the penumbra, but reaches values $>1.5$ in the outer penumbra.

\section{Discussion and conclusions}

We have investigated the relationship between the temperature and the magnetic field vector in a simple sunspot by employing parameters obtained from the inversion of 
spectropolarimetric data obtained in two neighboring $\mathrm{Fe}$ I lines (15648.5 $\AA$, and $15652.8 \AA$ ) in the IR $H$-band, for a simple sunspot. In order to compute the temperature, we use the continuum intensity values corrected for the non-magnetic stray light contamination. Thus our results are expected to be relatively unaffected by stray light. Overall, our results confirm the findings by earlier investigators (Kopp \& Rabin 1992; Solanki et al. 1993; Martínez Pillet \& Vázquez 1993; Stanchfield et al. 1997; Westendorp Plaza et al. 2001; Penn et al. 2002, 2003).

We found a nonlinear relationship between the field strength and temperature. We observe a strong variation of field strength (above $2800 \mathrm{G}$ ) with temperature and a more gradual change in field with temperature from 2400-2800 G; this includes the umbra-penumbra transition region. Below $2400 \mathrm{G}$, a steep change in field strength with temperature is observed. This relation is very similar to the relation obtained by Kopp \& Rabin (1992), Solanki et al. (1993) and Stanchfield et al. (1997). Recently, Penn et al. (2003) reported the results from observations of a sunspot using the Fe I $15648.5 \AA$ line. They also find a similar non-linear relationship between temperature and field strength. The difference in temperature between our results and theirs could be due to the use of two different continuum temperatures used for converting the spot brightness temperature.

The vertical field component follows a similar trend as the field strength, except that it does not increase as rapidly as $B$ with decreasing temperature. The radial magnetic component $B_{r}$ exhibits a more linear, but rather noisy relationship in the umbra.

Looking at Eqs. (5) or (6) it is clear that the spot temperature is a relatively complicated function of the magnetic field configuration. For a purely vertical homogeneous field, the equation can be interpreted as a linear relationship between $B^{2}$ and $T$, as long as the $B$ and $T$ values all refer to a fixed height and the ratio $m(r, z) / \rho(r, z)$ as well as the curvature force remains constant. Obviously, these conditions are approximately fulfilled in the inner umbra. Around the umbral boundary, however, the change in the thermal-magnetic relationship implies that there is a substantial departure from these conditions. Assuming the curvature force to be constant we obtain a notable jump of around $200 \mathrm{~km}$ in the Wilson depression at that location. In the penumbra the relation between $T$ and $B_{z}$ is steeper basically because the radiation is coming from a different height where the constant of proportionality,

$$
\frac{1}{8 \pi} \frac{T_{0}(z)}{P_{0}(z)} \frac{m(r, z)}{m_{0}(z)} \frac{\rho_{0}(z)}{\rho(r, z)},
$$

has a different value.

The relationship between magnetic field strength and line core intensity differs from the above especially for the penumbral points, where for Fer 15648.5 A line, we found two branches. I.e. the profiles of this line follow somewhat different relationships. These correspond to two sets of line-of-sight inclinations for the limb-side and center-side penumbra.

We also found a nonlinear relationship between the field inclination and temperature, especially in the outer penumbra, where a steep increase in field inclination is found for small change in temperature. Interestingly, this relationship looks like a mirror image of the $B$ vs. $T$ and $B_{z}$ vs. $T$ relationship. This differers from the linear relationship found by Solanki et al. (1993). An approximate linear relationship is found between the magnetic field strength and field inclination. This relationship is comparable with the results obtained by Stanchfield et al. (1997) and Westendorp Plaza (2001).

We have computed the Wilson depression at every point in the observed sunspot, thereby producing the first map of this quantity. In the umbra we found an average value of around $400 \mathrm{~km}$ and in the penumbra around $120 \mathrm{~km}$. While computing the Wilson depression we neglected the effect of curvature forces. The values obtained here confirm the results obtained using a similar method and similar data set by Solanki et al. (1993). Across the umbral-penumbral boundary a jump in $Z_{\mathrm{W}}$ of around $200 \mathrm{~km}$ is observed. This qualitative radial dependence of $Z_{\mathrm{W}}$ agrees with the picture obtained by utilizing the the Wilson effect (Wilson \& Cannon 1968; Wilson \& McIntosh 1969; Wittmann \& Schröter 1969). The difference in $Z_{\mathrm{W}}$ derived here (around $400 \mathrm{~km}$ in the umbra) and obtained from the Wilson effect $(600 \pm 200 \mathrm{~km}$, Gokhale \& Zwaan 1972) could be due to the effect of field-line curvature. The influence of curvature force on the derivation of the Wilson depression is discussed by Martínez Pillet \& Vázquez (1993) and Solanki et al. (1993). Considering the values of $Z_{\mathrm{W}}$ obtained from Wilson effect observations, i.e. taking 400 and $800 \mathrm{~km}$ as the lower and upper limits in the umbra (cf. Solanki et al. 1993) we computed values for $F_{\mathrm{c}} / 8 \pi$ in the umbra between 0 and $1.3 \times 10^{6} \mathrm{dyn}^{-2}$, with a most probable value of $5.8 \times 10^{5} \mathrm{dyn} \mathrm{cm}^{-2}$, corresponding to $Z_{\mathrm{W}}$ of $600 \mathrm{~km}$. This is well in line with the earlier values obtained for the same by Martínez Pillet \& Vázquez (1993) and Solanki et al. (1993). Since the Wilson effect measurements only provide a single, average umbral Wilson effect value it is not possible to determine the distribution of $F_{\mathrm{c}}$ over the whole sunspot. It is thus not possible to distinguish between the sunspot models, such as that of Jahn (1989), which has currents distributed beneath the whole penumbra, and Jahn \& Schmidt (1994), in which all currents are restricted to current sheets at the umbral and sunspot boundary.

In the umbra and in the inner penumbra, we found the gas pressure to be lower than the magnetic pressure. The plasma $\beta$ is lower than unity in the umbra and in most of the inner penumbra, but increases to values above 1.5 in the outer penumbra.

\section{References}

Alfvén, H. 1943, Arkiv. Mat. Astron. Fysik, 29, 11

Ballesteros, E., Collados, M., Bonet, J. A., et al. 1996, A\&AS, 115, 353

Balthasar, H., \& Schmidt, W. 1994, A\&A, 290, 649

Collados, M. 1999, in Magnetic field and oscillations, ed. B. Schmieder, B. Hofmann, \& J. Staude, ASP Conf. Ser., 184, 3

Dicke, R. H. 1970, ApJ, 159, 25

Frutiger, C. 2000, Inversion of Zeeman split Stokes profiles: Application to solar and stellar surface structures, Ph.D. Thesis, Institute of Astronomy, ETH Zürich, No. 13896 
Frutiger, C., Solanki, S. K., Fligge, M., \& Bruls, J. H. M. J. 2000, A\&A, 358, 1109

Gingerich, O., Noyes, R. W., Kalkofen, W., \& Cuny, Y. 1971, Sol. Phys., 18, 347

Gokhale, M. H., \& Zwaan, C. 1972, Sol. Phys., 26, 52

Jahn, K. 1989, A\&A, 222, 264

Jahn, K., \& Schmidt, H. U. 1994, A\&A, 290, 295

Kopp, G., \& Rabin, D. 1992, Sol. Phys., 141, 253

Maltby, P. 1977, Sol. Phys., 55, 335

Maltby, P., Avertt, E. H., Carlsson, M., et al. 1986, ApJ, 306, 284

Martínez Pillet, V., \& Vázquez, M. 1993, A\&A, 270, 494

Martínez Pillet, V., Collados, M., Sánchez Almeida, J., et al. 1999, in High Resolution Solar Physics: Theory, Observations, and Techniques, ed. T. R. Rimmele, K. S. Balasubramaniam, \& R. R. Radick, ASP Conf. Ser., 183, 264

Mathew, S. K., Lagg, A., Solanki, S. K., et al. 2003, A\&A, 410, 695 (Paper I)

Penn, M. J., Ceja, J. A., Bell, E., Frye, G., \& Linck, R. 2002, Sol. Phys., 205, 53
Penn, M. J., Walton, S., Chapman, G., Ceja, J., \& Plick, W. 2003, Sol. Phys., 213, 55

Press, W. H., Teukolsky, S. A., Vetterling, W. T., \& Flannery, B. P. 1992, Numerical Recipes (New-York: Cambridge Univ. Press)

Ruiz Cobo, B., \& del Toro Iniesta, J. C. 1992, ApJ, 398, 375

Solanki, S. K. 1987, Photspheric layers of solar magnetic fluxtubes, Ph. D. Thesis, Institute of Astronomy, ETH Zürich, No. 8309

Solanki, S. K. 2003, A\&AR, 11, 153

Solanki, S. K., Ruedi, I., \& Livingston, W. 1992, A\&A, 263, 312

Solanki, S. K., Walther, U., \& Livingston, W. 1993, A\&A, 277, 639

Spruit, H. C. 1974, Sol. Phys., 34, 277

Stanchfield II, D. C. H., Thomas, J. H., \& Lites, B. W. 1997, ApJ, 477, 485

Westendorp Plaza, C., del Toro Iniesta, J. C., Ruiz Cobo, B., et al. 2001, ApJ, 547, 1130

Wilson, P. R., \& Cannon, C. J. 1968, Sol. Phys., 4, 3

Wilson, P. R., \& McIntosh, P. S. 1969, Sol. Phys., 10, 370

Wittmann, A., \& Schröter, E. H. 1969, Sol. Phys., 10, 357 